Wilfrid Laurier University

Scholars Commons @ Laurier

Physics and Computer Science Faculty

Publications

Physics and Computer Science

1980

\title{
Voltage Locking and Other Interactions in Coupled Superconducting Weak Links. I. Theory
}

\author{
M.A.H. Nerenberg \\ University of Western Ontario \\ James A. Blackburn \\ Wilfrid Laurier University, jabjabjab@cogeco.ca \\ D.W. Jillie \\ Sperry Research Center
}

Follow this and additional works at: https://scholars.wlu.ca/phys_faculty

\section{Recommended Citation}

Nerenberg, M.A.H.; Blackburn, James A.; and Jillie, D.W., "Voltage Locking and Other Interactions in Coupled Superconducting Weak Links. I. Theory" (1980). Physics and Computer Science Faculty Publications. 56.

https://scholars.wlu.ca/phys_faculty/56

This Article is brought to you for free and open access by the Physics and Computer Science at Scholars Commons @ Laurier. It has been accepted for inclusion in Physics and Computer Science Faculty Publications by an authorized administrator of Scholars Commons @ Laurier. For more information, please contact scholarscommons@wlu.ca. 


\title{
Voltage locking and other interactions in coupled superconducting weak links. I. Theory
}

\author{
M. A. H. Nerenberg \\ Department of Applied Mathematics, University of Western Ontario, London, Ontario, Canada \\ James A. Blackburn \\ Physics Department, Wilfrid Laurier University, Waterloo, Ontario, Canada \\ D. W. Jillie \\ Sperry Research Center, Sudbury, Massachusetts 01776 \\ (Received 26 April 1979)
}

\begin{abstract}
A perturbation calculation is shown to give a satisfactory analytical description of the dc voltage characteristics of a coupled pair of superconducting weak links. In particular it predicts locking intervals over which the individual voltages of the junctions will either be equal or integer multiples of each other. Numerical simulations corroborate the perturbation approach and, as well, reveal the phenomenon of phase slippage between the junctions.
\end{abstract}

\section{INTRODUCTION}

The behavior of superconducting weak links has come to be fairly well understood during the past few years. It has been possible to predict current-phase relationships ${ }^{1}$ and so relate the family of Josephsontype devices: microbridges, point contact, and tunneling junctions. Various studies ${ }^{2,3}$ have investigated equivalent circuits, and it has been found that thinfilm microbridges can be represented adequately by a pure Josephson element with sinusoidal currentphase dependence, shunted by a resistance. ${ }^{4}$ This so-called RSJ model has been employed successfully in simulations of both thin-film interferometers ${ }^{5}$ and superconducting logic circuits. ${ }^{6}$ With the advent of the latter category of devices, the objective of high density has come to the fore. Thus it becomes essential to understand not only the properties of isolated, individual weak links, as discussed above, but also their behavior when located in close proximity to one another.

Recently Varmazis et al. ${ }^{7}$ reported studies of microbridges in which coupling was forced by the addition of gold shunt resistors to the samples. The radiated power from such weak-link pairs was shown to reflect coherence in the system; that is, the total power output was typically four times that of a single bridge, while the bandwidth was narrowed by a factor of 2 . It is also noteworthy that these results were found to be independent of the sense (relative directions) of the bias currents.

In this paper we examine theoretically the properties of such resistively shunted weak-link pairs, with particular emphasis on the appearance of phase coherence and voltage locking in the characteristics. The experimental situation is reviewed in Paper II, which deals extensively with nonshunted proximitycoupled links. The equations governing this latter arrangement are, however, formally the same as those for the system to be discussed here. Thus we choose for the moment to view the interaction as being induced by a shunt resistance, although other mechanisms can be accomodated.

\section{COUPLED WEAK LINKS}

We assume a series-connected pair of microbridges with critical currents $\left(i_{c 1}, i_{c 2}\right)$, phases $\left(\phi_{1}, \phi_{2}\right)$, and resistances $\left(R_{1}, R_{2}\right)$. The system is externally shunted by $R_{s}$ and, as indicated in Fig. 1, is biased by currents $\left(i_{1}, i_{2}\right)$. We suppose a sinusoidal currentphase relationship for each link, and also we presume $\dot{\phi}=2 \mathrm{eV} / \hbar$ for each device.

For convenience we define

$$
V_{0}=\frac{\overline{i_{c}} R_{1}\left(R_{2}+R_{s}\right)}{\left(R_{1}+R_{2}+R_{s}\right)}
$$

and employ normalized time $t^{*}=\left(2 e V_{0} / \hbar\right) t$ and indicate derivatives of phases in this dimensionless time with a $\left(^{*}\right)$ rather than a $(\cdot), \bar{i}_{c}$ is the average of $i_{c 1}$ and $i_{c 2}$, the Josephson zero-voltage current amplitudes. The resulting equations will now involve currents measured in units of $\bar{i}_{c}$. It is easy to show that, for the series-opposing case illustrated in Fig. 1,

$$
\begin{aligned}
& \stackrel{\phi_{1}}{=}=I_{1}-I_{c 1} \sin \phi_{1}+\alpha\left(I_{2}-I_{c 2} \sin \phi_{2}\right), \\
& \ddot{\phi}_{2}^{*}=\delta\left(I_{2}-I_{c 2} \sin \phi_{2}\right)+\alpha\left(I_{1}-I_{c 1} \sin \phi_{1}\right),
\end{aligned}
$$




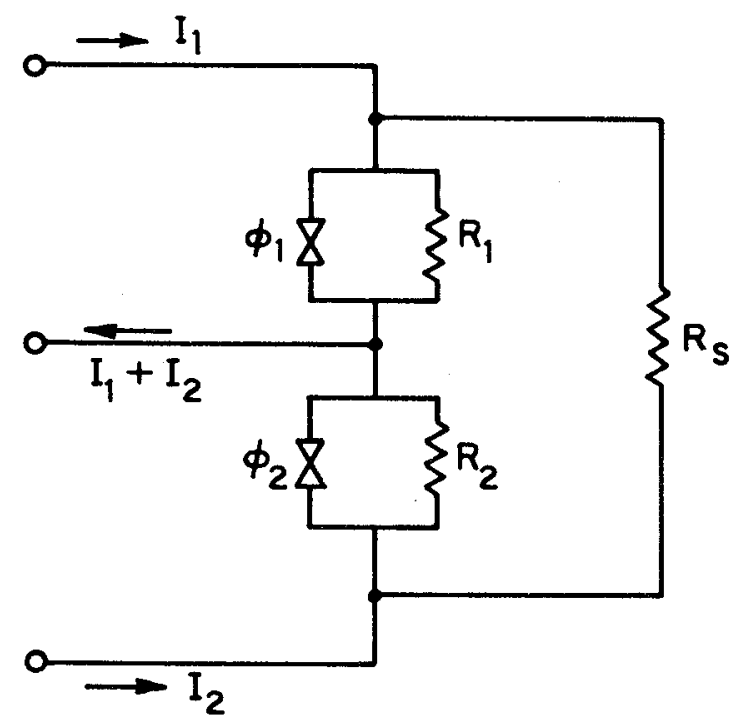

FIG. 1. Equivalent-circuit representation for two RSJ weak links coupled by an external shunt resistance.

where

$$
\begin{aligned}
& \alpha=\left(1+R_{s} / R_{2}\right)^{-1}, \\
& \delta=\frac{\left(1+R_{s} / R_{1}\right)}{\left(1+R_{s} / R_{2}\right)},
\end{aligned}
$$

and

$$
I_{1}=\frac{i_{1}}{\bar{i}_{c}}, \quad I_{2}=\frac{i_{2}}{\overline{i_{c}}}, \quad I_{c 1}=\frac{i_{c 1}}{\bar{i}_{c}}, \quad I_{c 2}=\frac{i_{c 2}}{\overline{i_{c}}} .
$$

The voltages $\dot{\phi}_{1}$ and $\dot{\phi}_{2}$ are, by convention in this configuration, measured across the links in opposite directions outward from the common midpoint.

For the series-aiding case, one of the bias currents in Fig. 1 must be reversed in direction. Now if we measure the link voltages $\dot{\phi}_{1}, \dot{\phi}_{2}$ in the same sense, then it can be shown that

$$
\begin{aligned}
& \dot{\phi}_{1}=I_{1}-I_{c 1} \sin \phi_{1}-\alpha\left(I_{2}-I_{c 2} \sin \phi_{2}\right), \\
& \dot{\phi}_{2}=\delta\left(I_{2}-I_{c 2} \sin \phi_{2}\right)-\alpha\left(I_{1}-I_{c 1} \sin \phi_{1}\right) .
\end{aligned}
$$

It should be noted that, mathematically, the seriesaiding case can be obtained from the opposing case by reversing the sign of $\alpha$, and vice versa.

The problems represented by the pairs of

Eqs. (1)-(4) were solved in two ways: a perturbation approach and a direct numerical simulation of these systems.

The perturbation approach has the advantage of determining analytically, as a function of the various parameters, the characteristics of the system. In particular, it predicts a "frequency-locking" region as well as the concomitant rapid change of the locked dc voltage in this region. The computational time using the perturbation result to determine the dc-voltage characteristics was miniscule compared to that required for the numerical simulation, which required long-time runs to determine a reliable dc voltage. The perturbation approach is limited, however, by being only approximate and having some difficulty in giving as accurate results just outside the frequencylocked region as it does in the other regions.

\section{PERTURBATION CALCULATION}

To be specific, we consider the series-aiding case: the opposing case being obtained from this one by changing the sign of $\alpha$. Making reasonable assumptions about the relative values of $R_{s}, R_{1}, R_{2}, I_{c 1}$, and $I_{c 2}$ would lead us to treat the coupling between $\phi_{1}$ and $\phi_{2}$ in Eqs. (3) and (4) as a perturbation of the uncoupled system $\left(R_{s} \rightarrow \infty\right.$; i.e., $\left.\alpha \rightarrow 0\right)$. A perturbation expansion using $\alpha$ as the "small" parameter was therefore carried out.

$A$ priori, such an expansion may be expected to be not very good as the equations are not approximately linear, and we require the knowledge of $\phi_{1}$ and $\phi_{2}$ for very large $t^{*}$, in principle in the limit as $t^{*} \rightarrow \infty$. This latter problem is mitigated, however, by the fact that we are primarily interested in the dc voltages, viz.

$$
\lim _{T \rightarrow \infty} \frac{\phi_{1}(T)}{T}
$$

and

$$
\lim _{T \rightarrow \infty} \frac{\phi_{2}(T)}{T}
$$

so that errors in $\phi_{1}$ and $\phi_{2}$ tend to be squelched in the voltages, at least provided they at worst grow linearly with time with only small coefficients.

We therefore write $\phi_{1}$ and $\phi_{2}$ as expansions in $\alpha$.

$$
\begin{aligned}
& \phi_{1}=\sum_{n=0}^{\infty} \alpha^{n} f_{n}(\alpha, t), \\
& \phi_{2}=\sum_{n=0}^{\infty} \alpha^{n} g_{n}(\alpha, t),
\end{aligned}
$$

where $f_{n}$ and $g_{n}$ can be shown from the resulting differential equations to be analytic at $\alpha=0$, thus ensuring the asymptotic nature of these expansions. Substituting into Eqs. (3) and (4), we obtain the differential equations that $f_{0}, f_{1}, g_{0}$, and $g_{1}$ satisfy

$$
\begin{aligned}
& f_{0}=I_{1}-I_{c 1} \sin f_{0}, \\
& g_{0}^{*}=\delta I_{2}-\delta I_{c 2} \sin g_{0},
\end{aligned}
$$

as the zeroth-order pair of equations, and

$$
\begin{aligned}
& f_{1}+\left(I_{c 1} \cos f_{0}\right) f_{1}=I_{c 2} \sin g_{0}-I_{2}, \\
& \stackrel{*}{g}_{1}+\left(\delta I_{c 2} \cos g_{0}\right) g_{1}=I_{c 1} \sin f_{0}-I_{1},
\end{aligned}
$$


as the pair of equations governing the first-order coirection. Equations (7) can be solved analytically, as in turn can Eqs. (8). However the latter equations predict an oscillating average $\tilde{f}_{1}$ and $\stackrel{g}{g}_{1}^{*}$ in the limit as $t^{*} \rightarrow \infty$.

To overcome this, a "renormalization" can be carried out self-consistently so that

$$
\lim _{t^{*} \rightarrow \infty} \frac{f_{1}\left(t^{*}\right)}{t^{*}}=0=\lim _{t^{*} \rightarrow \infty} \frac{g_{1}\left(t^{*}\right)}{t^{*}} .
$$

To make this renormalization process clear, Eqs. (3) and (4) are rewritten, adding and subtracting a constant term.

$$
\begin{aligned}
& \ddot{\phi}_{1}^{*}=\omega_{1}-I_{c 1} \sin \phi_{1}+\alpha\left(I_{c 2} \sin \phi_{2}-I_{2}-K\right), \\
& \dot{\phi_{2}}=\omega_{2}-\delta I_{c 2} \sin \phi_{2}+\alpha\left(I_{c 1} \sin \phi_{1}-I_{1}-L\right)
\end{aligned}
$$

where $\omega_{1}=I_{1}+\alpha K$ and $\omega_{2}=\delta I_{2}+\alpha L . \quad K$ and $L$ are constants ultimately to be chosen to make Eq. (9) true. The new versions of Eqs. (7) and (8) are, respectively,

$$
\begin{aligned}
& f_{0}^{*}=\omega_{1}-I_{c 1} \sin f_{0}, \\
& g_{0}^{*}=\omega_{2}-\delta I_{c 2} \sin g_{0},
\end{aligned}
$$

and

$$
\begin{aligned}
& f_{1}^{*}+\left(I_{c 1} \cos f_{0}\right) f_{1}=I_{c 2} \sin g_{0}-I_{2}-K, \\
& \stackrel{*}{g}_{1}+\left(\delta I_{c 2} \cos g_{0}\right) g_{1}=I_{c 1} \sin f_{0}-I_{1}-L .
\end{aligned}
$$

This "renormalization" is crucial in displaying frequency locking and in giving excellent agreement in general with the numerical simulation of the exact equations.

The nonlinear equations (12) are easily solved and give

$f_{0}\left(t^{*}\right)=2 \tan ^{-1} \frac{1}{\omega_{1}}\left[I_{c_{1}}+\Omega_{1} \tan \left(\frac{\Omega_{1} t^{*}}{2}+\psi_{1}\right)\right]$,

$g_{0}\left(t^{*}\right)=2 \tan ^{-1} \frac{1}{\omega_{2}}\left[\delta I_{c_{2}}+\Omega_{2} \tan \left(\frac{\Omega_{2} t^{*}}{2}+\psi_{2}\right)\right]$,

where

$$
\begin{aligned}
& \Omega_{1}=\left(\omega_{1}^{2}-I_{c 1}^{2}\right)^{1 / 2}, \\
& \Omega_{2}=\left(\omega_{2}^{2}-\delta^{2} I_{c 2}^{2}\right)^{1 / 2}, \\
& \psi_{1}=\tan ^{-1}\left(\frac{\omega_{1} \tan \left[f_{0}(0) / 2\right]-I_{c_{1}}}{\Omega_{1}}\right),
\end{aligned}
$$

where $Z_{1}=I_{c 1} / \omega_{1}$, and $Z_{2}=\delta I_{c 2} / \omega_{2}$. Using Eq. (15) we get and

$$
\psi_{2}=\tan ^{-1}\left(\frac{\omega_{2} \tan \left[g_{0}(0) / 2\right]-\delta I_{c_{2}}}{\Omega_{2}}\right) .
$$

It follows that

$$
\sin f_{0}=\frac{I_{c 1}+\omega_{1} \sin \left(\Omega_{1} t^{*}+\theta_{1}\right)}{\omega_{1}+I_{c 1} \sin \left(\Omega_{1} t^{*}+\theta_{1}\right)}
$$

and

$$
\sin g_{0}=\frac{\delta I_{c 2}+\omega_{2} \sin \left(\Omega_{2} t^{*}+\theta_{2}\right)}{\omega_{2}+\delta I_{c 2} \sin \left(\Omega_{2} t^{*}+\theta_{2}\right)},
$$

where

$$
\theta_{1}=\tan ^{-1}\left(I_{c 1} / \Omega_{1}\right)+2 \psi_{1}
$$

and

$$
\theta_{2}=\tan ^{-1}\left(\delta I_{c 2} / \Omega_{2}\right)+2 \psi_{2} .
$$

It is clear from Eqs. (15) that $\sin f_{0}$ and $\sin g_{0}$, aside from a zero-frequency component, contain, respectively, only the (angular) frequencies $\Omega_{1}$ with its harmonics and $\Omega_{2}$ with its harmonics. As we shall see below, this implies that frequency (voltage) locking occurs when $\Omega_{1}=\Omega_{2}$, or when one of these frequencies is a harmonic of the other.

To determine $\Omega_{1}$ and $\Omega_{2}$ explicitly we need to evaluate $K$ and $L$. This is done as follows. The integrating factor for Eq. (13a) is

$$
\exp \left(\int I_{c 1} \cos f_{0} d t^{*}\right)
$$

Since $d t^{*}=\left(f_{0}^{*}\right)^{-1} d f_{0}$, and in view of Eq. (12a), the integrating factor becomes

$$
\exp \left(\int \frac{I_{c 1} \cos f_{0}}{\omega_{1}-I_{c 1} \sin f_{0}} d f_{0}\right)=\frac{1}{\omega_{1}-I_{c 1} \sin f_{0}} .
$$

Similarly for Eq. (13b) the integrating factor is

$$
\frac{1}{\omega_{2}-\delta I_{c 2} \sin g_{0}} \text {. }
$$

Using the integrating factors in Eqs. (13) to obtain expressions for $f_{1}$ and $g_{1}$, and then invoking Eq. (9), we arrive at the following conditions on $K$ and $L$ :

$$
\begin{aligned}
& \lim _{T \rightarrow \infty} \frac{1}{T} \int_{0}^{T} \frac{-K-I_{2}+I_{c 2} \sin g_{0}}{1-Z_{1} \sin f_{0}} d t^{*}=0, \\
& \lim _{T \rightarrow \infty} \frac{1}{T} \int_{0}^{T} \frac{-L-I_{1}+I_{c 1} \sin f_{0}}{1-Z_{2} \sin g_{0}} d t^{*}=0,
\end{aligned}
$$

$$
K=-I_{2}+I_{c 2}\left[\lim _{T \rightarrow \infty} \frac{1}{T} \int_{0}^{T} \frac{Z_{2}+\sin \left(\Omega_{2} t^{*}+\theta_{2}\right)}{1+Z_{2} \sin \left(\Omega_{2} t^{*}+\theta_{2}\right)} d t^{*}+\lim _{T \rightarrow \infty} \frac{1}{T} \int_{0}^{T} Z_{1}\left(\frac{Z_{2}+\sin \left(\Omega_{2} t^{*}+\theta_{2}\right)}{1+Z_{2} \sin \left(\Omega_{2} t^{*}+\theta_{2}\right)}\right) \sin \left(\Omega_{1} t^{*}+\theta_{1}\right) d t^{*}\right]
$$


The last term in this expression for $K$ vanishes except when

$$
\Omega_{1}=n \Omega_{2}, \quad n=1,2, \ldots,
$$

while in the corresponding expression for $L$,

$$
L=-I_{1}+I_{c 1}\left[\lim _{T \rightarrow \infty} \frac{1}{T} \int_{0}^{T} \frac{Z_{1}+\sin \left(\Omega_{1} t^{*}+\theta_{1}\right)}{1+Z_{1} \sin \left(\Omega_{1} t^{*}+\theta_{1}\right)} d t^{*}+\lim _{T \rightarrow \infty} \frac{1}{T} \int_{0}^{T} Z_{2}\left(\frac{Z_{1}+\sin \left(\Omega_{1} t^{*}+\theta_{1}\right)}{1+Z_{1} \sin \left(\Omega_{1} t^{*}+\theta_{1}\right)}\right) \sin \left(\Omega_{2} t^{*}+\theta_{2}\right) d t^{*}\right] .
$$

The last term vanishes except when

$$
\Omega_{2}=m \Omega_{1}, \quad m=1,2, \ldots .
$$

We will call the cases when either $\Omega_{1}=n \Omega_{2}$, or $\Omega_{2}=m \Omega_{1}, m$ and $n$ positive integers, "resonant". We see that both $K$ and $L$ will have nonvanishing extra terms when the frequency-equality condition $\Omega_{1}=\Omega_{2}$ is satisfied. These terms lead to abrupt changes in the dc-voltage characteristics as this condition is met. Similarly, in the other resonant cases we have extra terms (this time in either $K$ or $L$ but not both) that give rise to abrupt changes in the dc-voltage characteristics as the corresponding frequency condition is met.

We obtain in the nonresonant case, i.e., when $\Omega_{1} \neq \Omega_{2}$ and furthermore when neither is $\Omega_{1}$ a harmonic of $\Omega_{2}$ nor vice versa

$$
\begin{aligned}
& K=\frac{1}{\delta}\left(\omega_{2}-\Omega_{2}\right)-I_{2}, \\
& L=\omega_{1}-\Omega_{1}-I_{1} .
\end{aligned}
$$

In the resonant case we restrict ourselves to listing the effect of resonances only up to the third harmonic, as beyond that the changes in $K$ and $L$ become extremely small. When $\Omega_{2}=\Omega_{1}$

$$
\begin{aligned}
& K=\frac{\left(\omega_{2}-\Omega_{2}\right)}{\delta}\left(1+\frac{Z_{1}}{Z_{2}} \frac{\Omega_{2}}{\omega_{2}} \cos \left(\theta_{1}-\theta_{2}\right)\right)-I_{2}, \\
& L=\left(\omega_{1}-\Omega_{1}\right)\left(1+\frac{Z_{2}}{Z_{1}} \frac{\Omega_{1}}{\omega_{1}} \cos \left(\theta_{1}-\theta_{2}\right)\right)-I_{1} .
\end{aligned}
$$

When $\Omega_{1}=2 \Omega_{2}$

$$
\begin{aligned}
K=\frac{\left(\omega_{2}-\Omega_{2}\right)}{\delta}(1 & +\frac{Z_{1}}{Z_{2}^{2}} \frac{\Omega_{2}}{\omega_{2}^{2}}\left(\omega_{2}-\Omega_{2}\right) \\
& \left.\times \sin \left(\theta_{1}-2 \theta_{2}\right)\right)-I_{2}
\end{aligned}
$$

and $L$ is given by Eq. (20); when $\Omega_{1}=3 \Omega_{2}$

$$
\begin{array}{r}
K=\frac{\left(\omega_{2}-\Omega_{2}\right)}{\delta}\left(1-\frac{Z_{1}}{Z_{2}^{3}} \frac{\Omega_{2}}{\omega_{2}^{3}}\left(\omega_{2}-\Omega_{2}\right)^{2}\right. \\
\left.\quad \times \cos \left(\theta_{1}-3 \theta_{2}\right)\right)-I_{2}
\end{array}
$$

and $L$ is again given by Eq. (20). However, when $\Omega_{2}=2 \Omega_{1} K$ is given by Eq. (19) and

$$
\begin{aligned}
L=\left(\omega_{1}-\Omega_{1}\right)(1 & +\frac{Z_{2}}{Z_{1}^{2}} \frac{\Omega_{1}}{\omega_{1}^{2}}\left(\omega_{1}-\Omega_{1}\right) \\
& \left.\times \sin \left(\theta_{2}-2 \theta_{1}\right)\right)-I_{1},
\end{aligned}
$$

when $\Omega_{2}=3 \Omega_{1} K$ is again given by Eq. (19) and

$$
\begin{aligned}
L=\left(\omega_{1}-\Omega_{1}\right)(1 & -\frac{Z_{2}}{Z_{1}^{3}} \frac{\Omega_{1}}{\omega_{1}^{3}}\left(\omega_{1}-\Omega_{1}\right)^{2} \\
& \left.\times \cos \left(\theta_{2}-3 \theta_{1}\right)\right)-I_{1} .
\end{aligned}
$$

Having now found $K$ and $L$, we see from Eq. (14) that the dc voltages $V_{1}$ and $V_{2}$

$$
V_{1,2}=V_{0} \lim _{T \rightarrow \infty} \frac{1}{T} \int_{0}^{T} \frac{d \phi_{1,2}}{d t^{*}} d t^{*}
$$

become, to first order of perturbation theory

$$
\begin{aligned}
& V_{1}=V_{0} \Omega_{1}, \\
& V_{2}=V_{0} \Omega_{2} .
\end{aligned}
$$

Equations (27) and (28) give the dc-voltage characteristics across each circuit as functions, say, of $I_{1}$ and $I_{2}$ via the frequency functions $\Omega_{1}$ and $\Omega_{2}$. Since only a constant of proportionality distinguishes the voltages from the frequencies, we will use these terms interchangeably.

\section{VOLTAGE LOCKING}

If we think of $I_{1}$ as well as $I_{c 1}, I_{c 2}, R_{1}, R_{2}$, and $R_{s}$ as being constants, the two voltages $V_{1}$ and $V_{2}$ vary only with $I_{2}$. Putting $V_{1}=V_{2}$ therefore would seen at first glance to determine one (positive) value of $I_{2}$ at which this occurs. However, Eqs. (21) and (22) show that this is not the case, as another variable, $\cos \left(\theta_{1}-\theta_{2}\right)$, related to a fixed average relative phase of the two circuits, intervenes and allows the equation $\Omega_{1}=\Omega_{2}$, (and, therefore, $V_{1}=V_{2}$ ) to have solutions over an interval of $I_{2}$ corresponding to the interval $[-1,1]$ for $\cos \left(\theta_{1}-\theta_{2}\right)$. (This interval in $I_{2}$ 
nonetheless shrinks to a point when the circuits happen to be identical.)

The results indicate that one may envisage events in the following way when in the neighborhood of $\Omega_{1}=\Omega_{2}$. For $I_{2}$ sufficiently small $V_{2}<V_{1}$; then as $I_{2}$ is increased the voltage difference diminishes up to a certain point where a further arbitrarily small increase in $I_{2}$ abruptly allows the circuits to come into synchronization. That is, the requirement $\Omega_{1}=\Omega_{2}$ is suddenly satisfied because Eqs. (21) and (22) have become compatible with it. ${ }^{8}$ As $I_{2}$ is increased further, the junctions remain locked at changing frequencies (and hence voltages), corresponding to different fixed average phase differences until the other extreme of the interval $[-1,1]$ for $\cos \left(\theta_{1}-\theta_{2}\right)$ is reached; at this point synchronization ceases to be possible, and the circuits jump into a state having a finite difference in their frequencies (and hence their dc voltage). This is illustrated in a particular case in Fig. 2.

The reference in the above discussion to fixed average phase differences is based on the fact that the first-order terms have the property that

$$
\lim _{T \rightarrow \infty} \frac{f_{1}(T)}{T}=0=\lim _{T \rightarrow \infty} \frac{g_{1}(T)}{T} .
$$

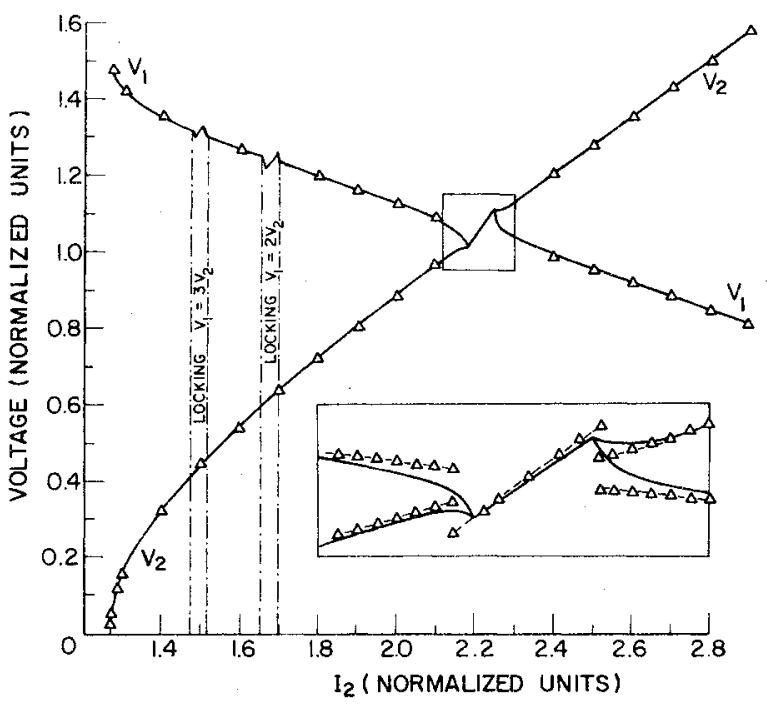

FIG. 2. Comparison of the voltage characteristics obtained from the numerical simulation of the exact equations with the perturbation calculation. Series-aiding case with: $I_{1}=2, I_{c 1}=1.2, I_{c 2}=0.8, \delta=\frac{2}{3}$, and $\alpha=0.2$. Inset: a blowup of the voltage-locked region and immediate environs when $\Omega_{1}=\Omega_{2}$. Triangles represent the results of the perturbation calculation, while the solid lines represent the numerical simulation. Where sufficient differences exist between the two calculations, dashed lines with triangles are used to represent the perturbation calculation. Voltages are in units of $V_{0}$.
But $f_{1}$ and $g_{1}$ are not identically zero; therefore, one would expect the junctions when frequency locked not to keep a fixed relative phase, but that the relative phase would oscillate around a fixed value determined by $\theta_{1}-\theta_{2}$. Indeed the numerical simulation of the exact equations described below corroborated this point.

Voltage locking when $V_{1}$ is a fixed multiple of $V_{2}$, or vice versa, will also occur when one of the frequencies $\Omega_{1}$ and $\Omega_{2}$ is a harmonic of the other. This harmonic frequency locking is evident from reasoning similar to that employed in the equal-frequency case, but applied to Eqs. (23)-(26). The appropriate function of the initial phases of the two circuits plays the role of creating a locking $I_{2}$ interval, and in determining together with the other parameters involved, both the extent of the interval and the voltage changes associated with the locking. We do not have to consider an infinite set of harmonics as the amplitude of the voltage change and the width of the locking interval diminish rapidly as the order of the harmonic increases. For the case considered in Fig. 2, the locking at $\Omega_{1}=2 \Omega_{2}$ is discernible and at $\Omega_{1}=3 \Omega_{2}$ is barely so on the scale utilized. Locking regions with $\Omega_{2}>\Omega_{1}$ are out of range.

It is no longer $\theta_{1}-\theta_{2}$ which maintains a fixed average value when the circuits are harmonically locked, but, for example, $\theta_{1}-2 \theta_{2}$ in the case $\Omega_{1}=2 \Omega_{2}$ and $\theta_{1}-3 \theta_{2}$ in the case $\Omega_{1}=3 \Omega_{2}$ which maintain fixed average values. This too was corroborated by the numerical simulation.

\section{NUMERICAL SIMULATION}

Equations (1) and (2) as well as Eqs. (3) and (4) were also solved numerically using Hamming's method, ${ }^{9}$ a predictor-corrector scheme with a truncation error $O\left(h^{5}\right)$, where $h$ is the step size. This method is well suited to the long-time runs required for ensuring accurate values of the dc voltages. In some cases runs of $10000 t^{*}$ units were used with an $h$ of 0.0625 in the same units. The accuracy was verified by halving the step size without finding significant change in the values of $\phi_{1}$ and $\phi_{2}$ at the end time. The use of a CDC Cyber 73 with its large word size undoubtedly aided in keeping roundoff error from being significant.

In most instances, including the frequency locked regions, a final $t^{*}$ of 2500 is more than adequate for determining stable values of the dc voltages. However, just outside the locked regions longer-time runs are required to achieve the same end. This phenomenon is illuminated somewhat by the perturbation approach, as it predicts that $\phi_{1}$ and $\phi_{2}$ will contain, near a frequency-locked region, admixtures of two nearly equal frequencies, which due to the nonlinearity of the systems lead to oscillation with a low difference frequency. 


\section{COMPARISON OF PERTURBATION THEORY WITH NUMERICAL SIMULATION}

In general there is excellent agreement between the perturbation calculation and the numerical simulation as shown in Fig. 2. The dc voltages, the positions, and widths of the intervals of frequency locking were all found to be in very close accord. The appropriate average phase difference as predicted by perturbation theory (related to $\theta_{1}-\theta_{2}$ when $\Omega_{1}=\Omega_{2}$, to $\theta_{1}-2 \theta_{2}$ when $\Omega_{1}=2 \Omega_{2}$, etc.) that corresponds to each $I_{2}$ in the locking intervals are corroborated extremely well by the numerical simulation.

The only area of disagreement one finds between perturbation "theory" and numerical "experiment" is just outside the frequency-locked regions. As mentioned above, and what is particularly visible in the inset of Fig. 2, the voltages are discontinuous in $I_{2}$ at the end points of the intervals of locking, according to perturbation theory. However the numerical simulation displays rapid but continous variation of the voltages when entering or leaving a locking interval.

Furthermore, in extreme proximity to the locking region, the numerical calculation demonstrates interesting behavior for $\phi_{1}$ and $\phi_{2}$ : when $I_{2}$ is near the interval where $\Omega_{1}=\Omega_{2}$, the relative phase $\left(\phi_{1}-\phi_{2}\right)$, averaged over suitable short-time intervals, changes very slowly when it is near 0 (modulo $2 \pi$ ) or $\pi$ (modulo $2 \pi$ ); the choice depends on which border of the locking interval one is at. Then after a lapse, during which this average remains essentially constant, it suddenly accelerates relatively rapidly until the average phase difference has changed by $2 \pi$. Once more it slows for a certain duration, after which

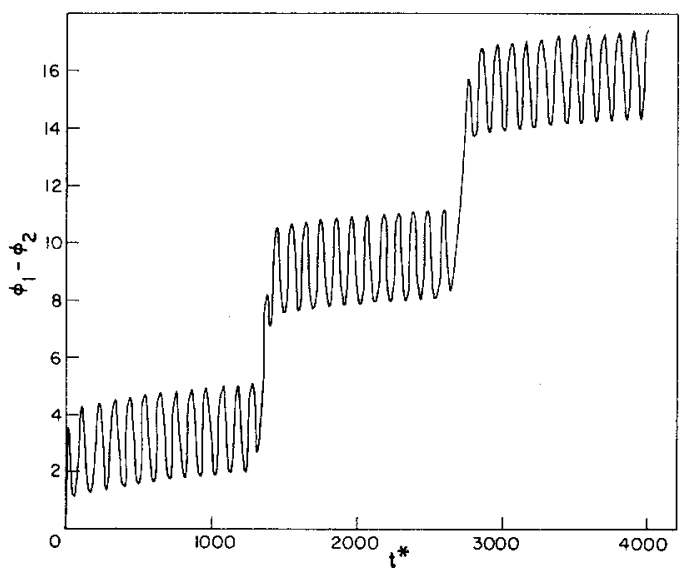

FIG. 3. Plot of $\left(\phi_{1}-\phi_{2}\right)$ as a function of $t^{*}$ for $0 \leqslant t^{*} \leqslant 4000$, as determined by the numerical simulation. The parameters $I_{1}, I_{c 1}, I_{c 2}, \delta$, and $\alpha$ are the same as for the case of Fig. 2; again this is the series-aiding case.

$I_{2}=2.17750$. See text for discussion.

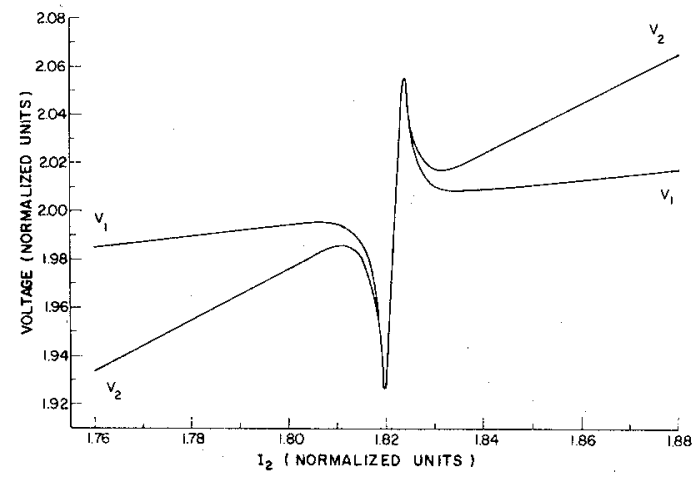

FIG. 4. Example of the voltage characteristics in the neighborhood of voltage locking when $\Omega_{1}=\Omega_{2}$, for the series-opposing case, obtained by numerical solution of the exact equations. $I_{1}=2.0, I_{c 1}=1.2, I_{c 2}=0.8, \delta=1$, and $\alpha=0.2$ were the parameters chosen. Voltages are in units of $V_{0}$.

it accelerates until the phase difference has again changed $2 \pi$, and so on. This is illustrated in Fig. 3, where $\left(\phi_{1}-\phi_{2}\right)$ is plotted against $t^{*}$, for the same junctions as are considered in Fig. 2. The $I_{2}$ chosen in Fig. 3 is just outside the locking interval.

As $I_{2}$ is changed to bring it in greater proximity to the locking interval, this phenomenon of relative stability followed by rapid "slip" by $2 \pi$ becomes more pronounced, until at one point for $I_{2}$ the average phase difference no longer slips, and the junctions are now locked.

This behavior might serve to explain the observations of Varmazis et al. ${ }^{7}$ and Sandell et al. ${ }^{10}$ of coherent radiation being emitted by such junctions without the latter being, in the dc-voltage locking state.

Similar behavior is observed when in the vicinity of the intervals in which there is harmonic locking of the dc voltages. There, rather than the average $\phi_{1}-\phi_{2}$ that stabilizes and then slips, it is the average $\phi_{1}-2 \phi_{2}$ when $\Omega_{1} \approx 2 \Omega_{2}$, and the average $\phi_{1}-3 \phi_{2}$ when $\Omega_{1} \approx 3 \Omega_{2}$, etc.; which behave in this fashion.

Finally, in Fig. 4 we present an example of voltage locking in the series-opposing case.

\section{SUMMARY}

A perturbation-theory approach was adopted to solve the problem of two coupled superconducting weak links. The results give insight into the behavior of such systems including the phenomenon of synchronization or voltage locking which is observed in the corresponding physical systems. A numerical simulation corroborated the results of the perturbation calculation, and complemented it by demonstrating a phase-slip phenomenon on the borders of the voltage-locking intervals. On the other hand, the 
perturbation calculation complemented the numerical simulation, for, without it as a guide, the intervals of locking might be overlooked, as in general they tend to be quite small.

The perturbation theory can be generalized to an extended system of coupled weak links, which the authors are currently carrying out. It is here that this approach is essential, for the computing time of the numerical simulation would be prohibitive for even an extended system of modest size.

\section{ACKNOWLEDGMENTS}

The authors thank Mr. David Meredith for assistance with the computer simulations. They are also grateful to J. E. Lukens and R. D. Sandell for helpful comments and for preprints of their work, and to $\mathrm{A}$. $S$. Deakin for extremely useful discussions on pertur bation expansions. This work was supported in part by grants from the National Science and Engineering Research Council of Canada.
${ }^{1}$ A. Baratoff, J. A. Blackburn, and B. B. Schwartz, Phys. Rev. Lett. 25, 1096, 1738 (1970).

2D. E. McCumber, J. Appl. Phys. 39, 3113 (1968).

${ }^{3}$ W. C. Stewart, Appl. Phys. Lett. 12, 277 (1968).

4P. E. Gregers-Hansen and M. T. Levinsen, Phys. Rev. Lett. 27, 847 (1971).

${ }^{5}$ C. D. Tesche and J. Clarke, J. Low Temp. Phys. 29, 301 (1977).

${ }^{6} \mathrm{H}$. W. Chan, W. Y. Lum, and T. Van Duzer, IEEE Trans. Magn. 11, 770 (1975).

${ }^{7}$ G. Varmazis, R. D. Sandell, A. K. Jain, and J. E. Lukens, Appl. Phys. Lett. 33, 357 (1978)
${ }^{8}$ Compare with discussion in reference to forced vacuum tube circuits, N. Minorsky, Non-Linear Oscillations (Van Nostrand, Princeton, 1962), Sec. 18.1.

${ }^{9}$ See for example M. L. James, G. M. Smith, and J. C. Wolford, Applied Numerical Methods for Digital Computations with Fortran and CSMP, 2nd ed. (Crowell, New York, 1977).

${ }^{10}$ R. D. Sandell, C. Varmazis, and J. E. Lukens, in Future Trends in Superconducting Electronics, edited by B. S. Deaver, C. M. Falco, J. H. Harris, and S. A. Wolf, AIP Conf. Proc. No. 44 (AIP, New York, 1979), p. 327. 